\title{
VARIABILIDAD ESPACIO-TEMPORAL DE LA TEMPERATURA DE SUPERFICIE EN ECOSISTEMAS DE DEHESA ESTIMADA MEDIANTE IMÁGENES LANDSAT TM: EL PAPEL DEL ARBOLADO
}

\author{
L. Vlassova \\ Facultad de Ciencias Ambientales \\ Universidad Técnica Estatal de Quevedo \\ Vía a Sto. Domingo km.1.5, EC120509, Quevedo, Ecuador \\ Ivlassova@uteq.edu.ec \\ Departamento de Geografía y Ordenación del Territorio-IUCA \\ Universidad de Zaragoza \\ Pedro Cerbuna, 12, 50006 Zaragoza \\ P. Rosero Tufiño \\ Facultad de Ciencias Agrarias \\ Universidad Técnica Estatal de Quevedo \\ Vía a Sto. Domingo km.1.5, EC120509, Quevedo, Ecuador \\ prosero@uteq.edu.ec

\section{R. Montorio Llovería} \\ Departamento de Geografía y Ordenación del Territorio-IUCA \\ Universidad de Zaragoza \\ Pedro Cerbuna, 12, 50006 Zaragoza \\ montorio@unizar.es
}

\begin{abstract}
Resumen: Las dehesas son sistemas agro-forestales en los que se producen complejos mecanismos de intercambio de carbono y agua debido a la presencia de estratos de vegetación con comportamiento eco-fisiológico contrastado: arbolado/herbáceo. Una de las variables clave en la parametrización del balance energético en estos ecosistemas es la temperatura de superficie (Ts). Este trabajo analiza su variación espacio-temporal en función de la cobertura arbórea en una dehesa al norte de Cáceres. La Ts se
\end{abstract}

Recibido: 16-10-2015. Aceptado: 28-10-2015. 
obtiene a partir de una serie de 14 imágenes Landsat-5 TM (2009-2011) que se agrupan en 3 compuestos estacionales (primavera, verano y otoño). La cobertura arbórea se estima a partir de ortofotografía e información del SIOSE. La distribución espacial de la Ts se relaciona con los niveles de cobertura en los compuestos de otoño y, especialmente, en verano momento en el que las diferencias medias entre las categorías extremas de arbolado $(<10 \%$ y $>60 \%)$ alcanzan $\operatorname{los} 2,5^{\circ} \mathrm{C}$.

Palabras clave: temperatura de superficie, cobertura arbórea, LANDSAT, dehesa.

\begin{abstract}
Dehesas are sylvo-agro-pastoral ecosystems with complex mechanisms of carbon and water interchange due to existence of contrasted ecophysiological vegetation layers: tree and grass. One of the key variables in parametrization of energy balance in these systems is Land Surface Temperature (LST). This study analyzes spatio-temporal variability of LST as a function of tree cover in dehesa ecosystem in the northern part of the province of Cáceres. LST is retrieved from a series of 14 Landsat-5 TM images (2009-2011) grouped and analyzed by season (spring, summer and autumn). Tree cover is estimated from the orthophotos and SIOSE data. Spatial distribution of LST is related to to the percentage of tree cover in autumn and even more in summer, the moment when mean differences among the lowest and the highest categories established according tree cover percentage $(<10 \%$ and $>60 \%)$ achieve $2,5^{\circ} \mathrm{C}$.
\end{abstract}

Keywords: land surface temperature, tree cover, LANDSAT, dehesa.

\title{
1. Introducción
}

El término dehesa refiere a un tipo de monte arbolado de uso agrosilvopastoril (San Miguel, 1994) resultado de un largo proceso de transformación (Blanco et al., 1997; Díaz et al., 1997). Principalmente se localiza en la parte suroccidental de la Península Ibérica, extendiéndose en España sobre una superficie de más de 3,5 millones de hectáreas (Olea et al., 2005). Su alto valor ecológico, socio-cultural y económico (Pulido et al., 2001; Montero et al., 1998) hace que estos hábitats estén incluidos en la Directiva 92/43 de la Unión Europea para su preservación. En este sentido, algunos estudios expresan dudas respecto a su sostenibilidad (Pulido et al., 2001 y Díaz et al., 1997) debido a los problemas derivados del sobrepastoreo, la intensificación del laboreo agrícola o la escasez de regeneración del arbolado (Moreno y Pulido, 2009). En relación con este último extremo, el predominio de los árboles de edad intermedia/avanzada y la ausencia de plantas jóvenes constituye uno de los problemas más importantes (Montero et al., 1998; Plieninger 
et al., 2004, entre otros). Según datos del Tercer Inventario Forestal Nacional (IFN3) en la provincia de Cáceres, la regeneración se considera insuficiente o nula en 2/3 de la superficie evaluada a nivel nacional (Pulido y Picardo, 2010).

La dehesa se caracteriza por su estructura en dos estratos: arbolado/matorral disperso y pastizal o cultivo. La fracción de cabida cubierta más habitual oscila entre el 5\% y el 60\% con una densidad promedio de 40-90 pies por hectárea que puede ser más baja (10) o más alta (200) dependiendo del tipo de dehesa (Huntsinger et al., 2013; Pulido et al., 2010; Carreiras et al., 2006). Las especies comúnmente encontradas en el estrato leñoso son Quercus ilex L. subsp. ballota, Quercus suber, Quercus faginea Lam. y diferentes arbustos perennifolios. El pastizal presenta un reducido potencial productivo que depende de la pluviometría. Entre los taxones más representativos de la fracción herbácea encontramos especies de gramíneas como Aira caryophyllea L., Airopsis tenella (Cav.) Asch. \& Graebn., Psilurus incurvus (Gouan) Schinz \& Tell y Bromus sp., algunos especies de Trifolium entre leguminosas, y Echium plantagineum L., Spergula arvensis L., Rumex acetosella L., Erygium campestre L. o Erodium cicutarium L. de otras familias (Devesa, 1995; Olea et al., 2005).

En las dehesas los mecanismos de intercambio de carbono y agua son complejos debido a la presencia de estratos de vegetación diferenciados desde el punto de vista ecofisiológico. En gran medida los ciclos de carbono y agua son controlados por los flujos de energía que son distintos en las copas de árboles y pastizal (Baldocchi et al., 2004). De esta manera, los estudios de balance de energía son necesarios para validación de los modelos de dehesa que buscan predecir estados de equilibrio entre vegetación y la humedad de suelo (Eagleson, 1982) y el efecto que puede ejercer en estos ecosistemas el cambio climático (Rodriguez-Iturbe et al., 1999; Volder et al., 2013; Zeng y Neelin, 2000).

La temperatura de superficie (Ts) constituye una de las claves en la modelización de los intercambios de energía, agua y carbono que se producen entre la vegetación, el suelo y la atmósfera, debido a su influencia sobre la tasa metabólica de las plantas y la descomposición de la materia orgánica del suelo, dos aspectos muy importantes en relación con la transferencia neta de carbono a la atmósfera (Miquelajauregui, 2013). A su vez, la presencia y, más específicamente, el tipo de vegetación juegan un rol importante en el control de la Ts, debido a las diferencias en emisividad entre los componentes bióticos y abióticos que conforman la dehesa (Quattrochi y Luvall, 2000).

Tanto la temperatura de la superficie (Ts) como la abundancia de la vegetación pueden ser analizadas a partir de la información proporcionada por sensores remotos, entre los cuales se destacan los datos de las misiones Landsat. Las imágenes Landsat- 5 TM (Thematic Mapper) han sido utilizadas profusamente en el estudio de la distribución espacio-temporal de vegetación a través de los diferentes índices espectrales a partir de la información captada en la región óptica del espectro electromagnético. El más ampliamente usado es el índice de vegetación de diferencia normalizada NDVI, que 
se relaciona con diferentes propiedades biofísicas de la vegetación (clorofila, cubrimiento, LAI, fAPAR, etc.) (Daughtry et al., 2000; Hwang et al., 2011; Turner et al., 1999). Las imágenes Landsat también poseen una banda en el infrarrojo térmico que posibilita la espacialización de la Ts (Vlassova et al., 2014; Weng et al., 2004).

El objetivo de este trabajo consiste en analizar la variabilidad espacio-temporal de la Ts, estimada a partir de las imágenes Landsat, en función de la densidad de arbolado en una zona de dehesa situada al norte de la provincia de Cáceres. La utilización conjunta de índices de vegetación procedentes de información multiespectral, de cartografía de la Ts y de información sobre las proporciones que ocupan diferentes estratos ofrece la posibilidad de crear modelos de la dehesa más precisos. Los resultados de estos modelos pueden ser integrados en el diseño de planes que aseguren la sostenibilidad y el mantenimiento de las funciones productivas y ecológicas (protección frente a la erosión, mantenimiento de la biodiversidad y regulación de los flujos de carbono y agua) de la dehesa (Moreno y Pulido, 2009).

\section{Material y métodos}

\section{1. Área de estudio}

La zona de estudio de $70 \mathrm{~km}^{2}$ se localiza en un ecosistema de dehesa en el noreste de la provincia de Cáceres (Figura 1), muy cerca del área de estudio del proyecto de investigación FLUXPEC (CGL2012-34383): "Seguimiento de flujos de agua y carbono mediante teledetección en ecosistemas mediterráneos de dehesa" (http://www.lineas.cchs. csic.es/fluxpec/) que incluye una torre de medición de flujos de agua y carbono por

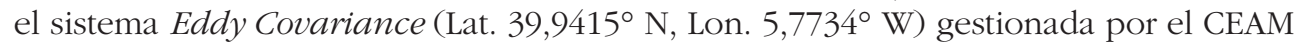
(Centro de Estudios Ambientales del Mediterráneo) desde el año 2003. El área se caracteriza por un clima típico mediterráneo con veranos secos y calurosos e inviernos húmedos y templados. La temperatura y precipitación media anual son $16,7^{\circ} \mathrm{C}$ y $572 \mathrm{~mm}$, respectivamente. La altitud media sobre el nivel del mar es de $256 \mathrm{~m}$. Las especies perennifolias de Quercus ilex L. subsp. Ballota y Quercus suber predominan en el estrato arbóreo y las de Trifolium glomeratum L., Echium plantagineum L., y Spergula arvensis son los más comunes en el pastizal.

\subsection{Imágenes de satélite}

El estudio se basa en una serie multitemporal de 14 imágenes Landsat-5 TM adquiridas sobre el área de estudio entre junio de 2009 y septiembre de 2011 (Tabla 1). Las imágenes georreferenciadas se descargaron del servidor del Servicio Geológico de los Estados Unidos o USGS por sus siglas en inglés (https://lpdaac.usgs.gov/get_data). 


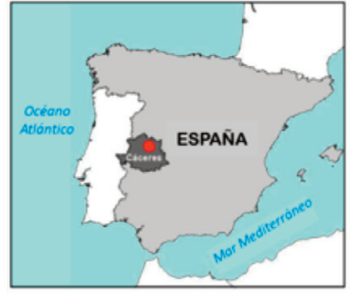

- Área de estudio

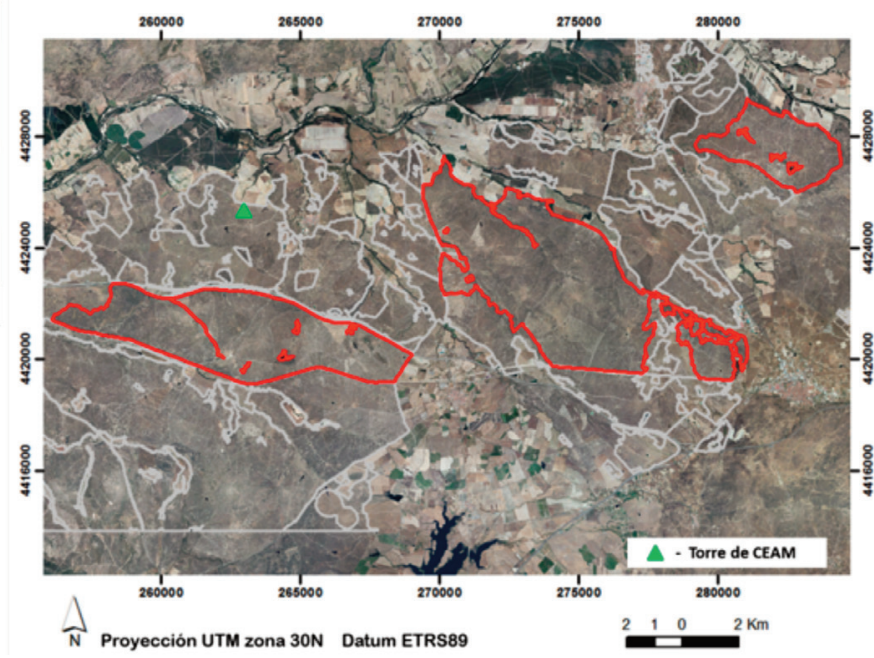

Figura 1. Ubicación y ortofotografía del área de estudio (límites en rojo). Las líneas de color gris claro delimitan el ecosistema de dehesa (Sistema de Información de Ocupación del Suelo en España, SIOSE).

Tabla 1. Fechas y condiciones de iluminación en el momento de adquisición de las imágenes utilizadas

\begin{tabular}{|l|c|c|c|c|}
\hline Fecha & Hora (UTC) & $\begin{array}{c}\text { Azimut solar } \\
\text { (grados) }\end{array}$ & $\begin{array}{c}\text { Elevación solar } \\
\text { (grados) }\end{array}$ & $\begin{array}{c}\text { Estación } \\
\text { climática }\end{array}$ \\
\hline 27-jun-09 & $10: 50: 18$ & 123,55 & 63,88 & verano \\
\hline 29-jul-09 & $10: 50: 49$ & 128,98 & 59,94 & verano \\
\hline 30-ago-09 & $10: 51: 18$ & 141,13 & 52,63 & verano \\
\hline 17-oct-09 & $10: 51: 53$ & 156,52 & 37,36 & otoño \\
\hline 10-mar-10 & $10: 52: 43$ & 146,85 & 40,13 & primavera \\
\hline 11-abr-10 & $10: 52: 40$ & 141,79 & 52,28 & primavera \\
\hline 30-jun-10 & $10: 52: 19$ & 124,31 & 64,00 & verano \\
\hline 16-jul-10 & $10: 52: 16$ & 126,06 & 62,26 & verano \\
\hline 01-ago-10 & $10: 52: 10$ & 130,34 & 59,61 & verano \\
\hline 05-nov-10 & $10: 51: 34$ & 159,16 & 31,40 & otoño \\
\hline 16-may-11 & $10: 51: 20$ & 132,51 & 61,79 & primavera \\
\hline 01-jun-11 & $10: 51: 13$ & 127,86 & 63,89 & verano \\
\hline 04-ago-11 & $10: 50: 41$ & 130,72 & 58,86 & verano \\
\hline 05-sep-11 & $10: 50: 24$ & 142,93 & 50,94 & otoño \\
\hline
\end{tabular}


En el trabajo se utilizaron las bandas ópticas en las regiones espectrales de rojo $(0,63-$ $0,69 \mu \mathrm{m})$ e infrarrojo cercano $(0,76-0,90 \mu \mathrm{m})$, con una resolución espacial de $30 \mathrm{~m}, \mathrm{y}$ la banda térmica $(10,4-12,5 \mu \mathrm{m})$, con una resolución de $120 \mathrm{~m}$.

Para la corrección atmosférica de las reflectividades se ha utilizado la herramienta LEDAPS (Landsat Ecosystem Disturbance Adaptive Processing System), basada en el código de transferencia radiativa 6S (Wolfe et al., 2004). El algoritmo emplea datos auxiliares (ozono, vapor de agua, grosor óptico de la atmósfera) para corregir en las imágenes las distorsiones causadas por la dispersión y absorción atmosférica. En el caso de los datos térmicos, los niveles digitales originales fueron transformados primero a valores de radiancia y luego en temperatura de brillo usando las constantes de calibración proporcionadas en los metadatos (Chander et al., 2009).

\subsection{Estimación de la temperatura de superficie (Ts) e índice de vegetación}

La temperatura de superficie (Ts) ha sido estimada mediante el método de monocanal (Jiménez-Muñoz et al., 2010) que, aparte de la emisividad de la superficie $\varepsilon$ requerida por cualquier algoritmo de Ts, sólo necesita el contenido de vapor en la atmósfera como dato auxiliar. La Ts se calcula usando la ecuación:

$$
T s=\gamma\left[\frac{1}{\varepsilon}\left(\psi_{1} L_{\text {sensor }}+\psi_{2}\right)+\psi_{3}\right]+\delta
$$

donde $\gamma$ and $\delta$ son parámetros calculados a partir de la radiancia $\left(L_{\text {sensor }}\right)$ y temperatura de brillo $\left(T_{\text {sensor }}\right)$ usando las expresiones:

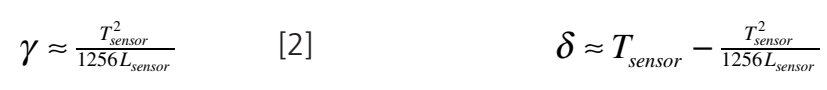

y $\psi 1, \psi 2, \psi 3$ son funciones atmosféricas, que para Landsat-5 TM vienen dadas por:

$$
\begin{aligned}
& \psi_{1}=0.14714 w^{2}-0.15583 w+1.1234 \\
& \psi_{2}=-1.1836 w^{2}-0.37607 w-0.52894 \\
& \psi_{3}=-0.04554 w^{2}+1.8719 w-0.39071
\end{aligned}
$$

donde $w$ es el contenido total del vapor en la atmósfera en $\mathrm{g}_{\mathrm{cm}}^{-2}$.

En este estudio se utilizaron los valores de vapor atmosférico de la base de datos del Centro Nacional para Análisis Atmosférico de EEUU (http://www.esrl.noaa.gov/psd/ data/). El método es capaz de estimar la Ts con un margen de error $\pm 1^{\circ} \mathrm{C}$ cuando el contenido de vapor atmosférico se encuentra en el rango de $0,5-2,5 \mathrm{~g} \mathrm{~cm}^{-2}$ (JiménezMuñoz et al., 2010). 
Para el análisis temporal de la vegetación se ha utilizado el índice de vegetación de diferencia normalizada NDVI (Rouse et al., 1974), que se basa en la diferencia de la reflectividad entre las bandas de las regiones espectrales rojo (banda 3) e infrarrojo cercano (banda 4) característica para la vegetación. El NDVI también se aplicó para estimar la emisividad por el método de umbrales de NDVI de Sobrino y Raissouni (2000), que asigna los valores de emisividad de acuerdo a los rangos de este índice.

\subsection{Cartografía de la distribución espacial de la densidad del arbolado}

Para la identificación de la dehesa se han utilizado los datos del Sistema de Información de Ocupación del Suelo en España (SIOSE) incluido en el Plan Nacional de Observación del Territorio en España (PNOT), coordinado por el Instituto Geográfico Nacional (IGN). La información consiste en capas de polígonos georeferenciados en proyección UTM (huso 30N, datum ETRS89) con escala de referencia 1:25000 (http:// www.siose.es). A partir de la cobertura 701 dehesa (código DHS) se ha extraído la información referente al porcentaje de cubrimiento de frondosas caducifolias (FDC), frondosas perennifolias (FDP), matorral (MTR) y pastizal (PST). Dentro de la zona de estudio se han seleccionado para su análisis los tres polígonos más extensos cuya fracción leñosa (arbolado y matorral) se encuentra en el rango de 30-50\% característico de la dehesa (Figura 1).

Dentro de los polígonos seleccionados se ha analizado la variabilidad espacial del arbolado mediante el uso de la ortofotografía digital generada en el marco del PNOT a partir de los datos de junio 2012. El fotomosaico (píxel de 0,25 m) correspondiente a la hoja 0624 del MTN50 (Mapa Topográfico Nacional 1:50.000) fue descargado del servidor del CNIG (http://centrodedescargas.cnig.es/CentroDescargas) en formato ECW, sistema geodésico de referencia ETRS89 y proyección UTM huso 30N. En la etapa de pre-procesamiento la imagen RGB fue convertida a escala de grises.

Las imágenes (Figura 2A) fueron clasificadas en dos categorías: vegetación leñosa de árboles/matorral (píxeles con niveles de gris menor de 130) y pastizal (píxeles con niveles de gris mayor o igual a 130). Como resultado de la clasificación se ha obtenido una nueva imagen ráster con píxeles de dos valores: 1 para "cobertura arbórea" y 0 para "pastizal" (Figura 2B). Tras ello, aplicando funciones de estadística zonal, se calculó la cantidad de píxeles de "cobertura arbórea" en píxeles Landsat y, finalmente, el porcentaje de cobertura arbórea en píxeles Landsat $\left(\mathrm{CA}_{\%}\right)$ a través de la expresión:

$$
C A_{\%}=\frac{n^{*} 100}{N}
$$

donde $n$ es la cantidad de píxeles con cobertura arbórea dentro del píxel Landsat y $N=$ 14400 es la cantidad de píxeles de ortofoto en un píxel de la imagen satelital. 

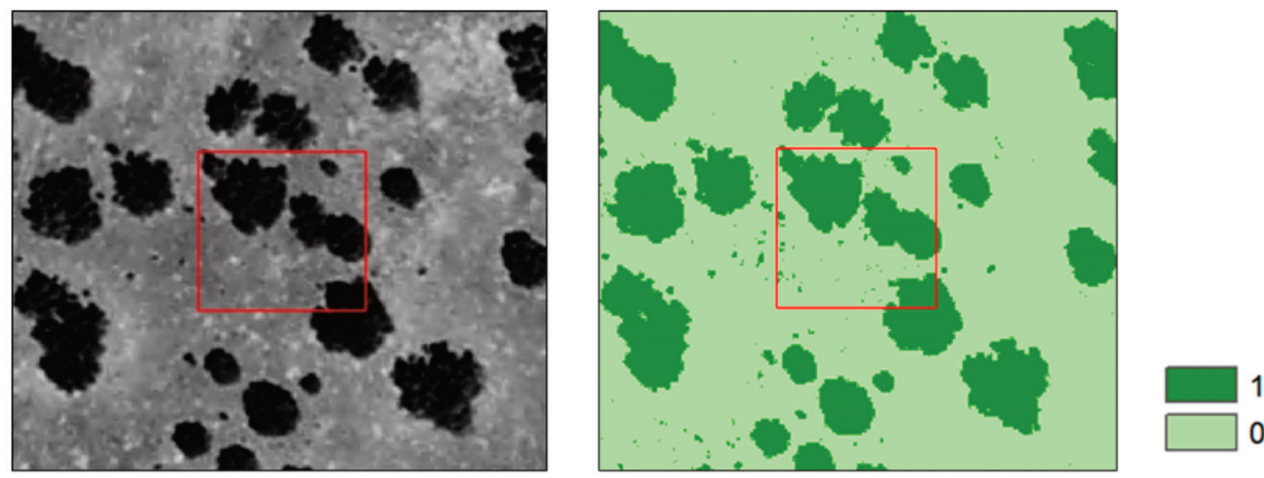

Figura 2. (A) Identificación de un píxel de Landsat-5 TM sobre la ortofotografía del PNOA; (B) Raster de clasificación de ortofotografía en "cobertura arbórea" (píxeles de valor 1) y "pastizal" (píxeles de valor 0).

\subsection{Análisis estadístico}

Se han establecido 7 categorías de dehesa en función del porcentaje de la cobertura arbórea: <10\%; 10-20\%; 20-30\%; 30-40\%; 40-50\%; 50-60\%; >60\%. La determinación de los intervalos se basó en dos criterios: (1) generar intervalos regulares de cobertura arbórea dentro del rango de mayor representación de estos ecosistemas, entre 10\% y 60\% (Moreno y Pulido, 2009; Pulido y Picardo, 2010); y (2) disponer de suficiente número de píxeles en cada categoría para hacer posible el equilibrio de las submuestras en el análisis estadístico. Para evitar la contaminación de los resultados por la influencia de las áreas adyacentes y de los componentes no propios de la dehesa, se han excluido del análisis los píxeles situados a menos de 120 m de los límites de polígonos, así como los pertenecientes a otras cubiertas diferentes a la dehesa (láminas de agua, cultivos, edificios). El análisis se concentró en los dos componentes estructurales de la dehesa: el estrato con presencia del material leñoso (árboles y matorral) y el estrato herbáceo.

En cada categoría se ha identificado una muestra aleatoria de 1000 puntos (píxeles de la imagen) que representan de manera equilibrada las 7 categorías de dehesa. Considerando los ciclos anuales de las variables climáticas y fenología del componente herbáceo de la dehesa, se han generado los compuestos de los datos correspondientes a los valores promedio de imágenes de cada estación climática (primavera, verano y otoño) (Tabla 1), realizándose la prueba de ANOVA y la prueba post hoc de Tamhane T2 para determinar la existencia de diferencias significativas de Ts y NDVI entre categorías. 


\section{Resultados y discusión}

\subsection{Cartografía de la cobertura arbórea}

La figura 3 representa la distribución espacial de los porcentajes de cobertura arbórea en el área de estudio. La clasificación digital de la ortofotografía permite identificar espacialmente el desigual reparto del arbolado en cada uno de los tres polígonos del SIOSE.



Cobertura arbórea $(\%)$

Figura 3. Distribución espacial de la cobertura arbórea en el área de estudio.

Los tonos marrones, correspondientes a las categorías en las que el recubrimiento del arbolado es intermedio (20-30\% y 30-40\%) son predominantes, ocupando en torno al 50\% de la superficie analizada, lo que coincide con las descripciones del SIOSE para estas unidades (30\% de cobertura arbórea y hasta 15\% de matorral). En el otro extremo, el color verde oscuro, que representa las zonas en las que la ocupación del arbolado es superior al 60\%, es poco representativo ( $3.5 \%$ de la superficie), siendo más profuso en el extremo occidental del polígono situado al Oeste y en zonas específicas del polígono que ocupa la posición central. En el polígono oriental los píxeles de esta categoría son casi inexistentes.

\subsection{Patrones de distribución espacio-temporal del NDVI y Ts}

La distribución espacial de la Ts y el NDVI correspondientes a los compuestos de primavera, verano y otoño se muestra en la figura 4. Los valores más elevados de NDVI (con un valor promedio de $\sim 0,57$ ) se observan en el compuesto de primavera, presentando un reparto bastante homogéneo sobre la superficie analizada. Sin embargo, a nivel térmico, se aprecia una mayor heterogeneidad espacial que se concreta en un gradiente moderado con dirección este-oeste. 


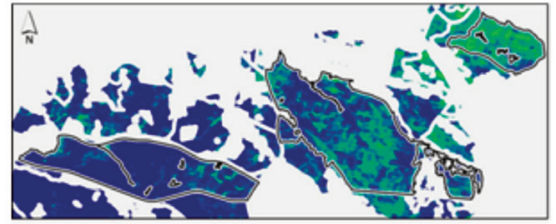

Temperatura_primavera



Temperatura_verano

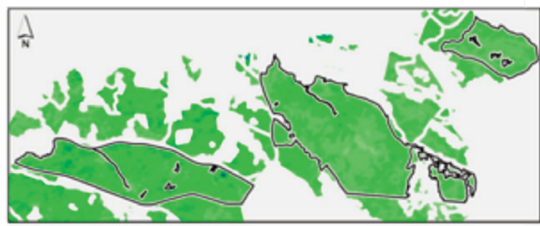

Temperatura_otoño

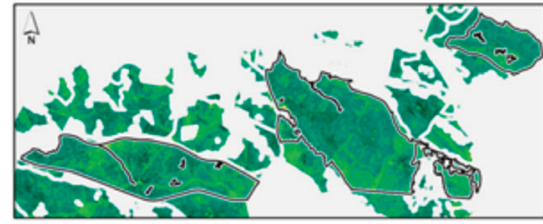

NDVI_primavera

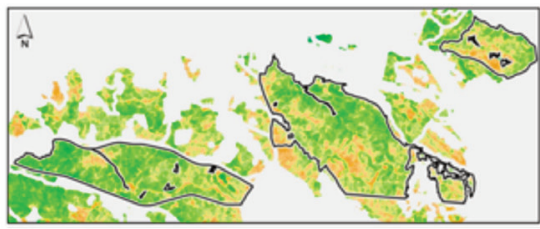

NDVI_verano



NDVI_otoño

Figura 4. Distribución de la Ts (izquierda) y NDVI (derecha) estimados a partir de las imágenes Landsat-5 TM en el área de estudio.

Los valores más bajos de NDVI, tal como se esperaba, se recogen en el compuesto de verano. Su distribución presenta una gran variabilidad espacial, de tal modo que se reconocen sectores específicos en los que los valores de NDVI alcanzan valores elevados $(\sim 0,5)$ y otros en donde los valores de NDVI se aproximan a 0 (núcleo del polígono central). Este patrón se reproduce a nivel térmico al observarse diferencias considerables entre zonas en las que los valores de NDVI también son reseñables. Los valores de Ts más elevados se observan en el núcleo del polígono central y, en el extremo contrario, las Ts más bajas se recogen en la zona occidental, reproduciendo groseramente la distribución de los valores de NDVI y la de los porcentajes de cobertura arbórea. El compuesto de otoño (parte inferior de la figura 4) representa espacialmente una situación intermedia entre las dos anteriormente descritas.

A nivel estadístico, la Tabla 2 y la Figura 5 (A-C) recogen, respectivamente, algunos indicadores descriptivos de la Ts y los intervalos de confianza para la media al 95\%, extraídos de las distintas categorías de dehesa para los tres compuestos (primavera, verano y otoño). En términos generales, se advierte una clara relación entre los valores de Ts y los niveles de densidad del arbolado, de tal modo que un aumento del porcentaje de 
Tabla 2. Estadísticos descriptivos de la Ts $\left({ }^{\circ} \mathrm{C}\right)$ por categorías de dehesa y estaciones climáticas

\begin{tabular}{|c|c|c|c|c|c|}
\hline & Categoria (\% de cobertura arbórea) & Media & Desviación típica & Mínimo & Máximo \\
\hline \multirow{7}{*}{ 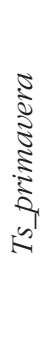 } & $<10$ & 22.10 & 0.77 & 19.01 & 23.86 \\
\hline & $10-20$ & 21.94 & 0.77 & 19.59 & 23.83 \\
\hline & $20-30$ & 21.79 & 0.82 & 19.52 & 23.85 \\
\hline & $30-40$ & 21.60 & 0.88 & 19.19 & 23.89 \\
\hline & $40-50$ & 21.45 & 0.88 & 18.88 & 23.89 \\
\hline & $50-60$ & 21.41 & 0.85 & 19.02 & 24.53 \\
\hline & $>60$ & 21.31 & 0.84 & 19.22 & 24.24 \\
\hline \multirow{7}{*}{$\begin{array}{l}\stackrel{2}{\vdots} \\
\vdots \\
\vdots \\
\vdots \\
n^{\prime}\end{array}$} & $<10$ & 42.11 & 1.03 & 38.48 & 44.38 \\
\hline & $10-20$ & 41.80 & 1.01 & 38.68 & 44.45 \\
\hline & $20-30$ & 41.46 & 1.06 & 38.14 & 44.35 \\
\hline & $30-40$ & 41.11 & 1.04 & 37.58 & 43.68 \\
\hline & $40-50$ & 40.76 & 1.10 & 37.43 & 43.59 \\
\hline & $50-60$ & 40.50 & 1.10 & 36.14 & 43.47 \\
\hline & $>60$ & 39.85 & 1.35 & 36.01 & 43.14 \\
\hline \multirow{7}{*}{  } & $<10$ & 26.26 & 0.76 & 23.96 & 28.22 \\
\hline & $10-20$ & 25.97 & 0.77 & 23.52 & 28.17 \\
\hline & $20-30$ & 25.68 & 0.79 & 23.45 & 28.12 \\
\hline & $30-40$ & 25.44 & 0.80 & 23.19 & 28.07 \\
\hline & $40-50$ & 25.18 & 0.80 & 23.07 & 27.56 \\
\hline & $50-60$ & 25.05 & 0.82 & 22.19 & 27.17 \\
\hline & $>60$ & 24.74 & 0.88 & 22.03 & 27.05 \\
\hline
\end{tabular}

la fracción leñosa conlleva una disminución en los valores de la Ts. Esta relación inversa es muy evidente en los compuestos de verano y otoño, recogiéndose diferencias estadísticamente significativas entre todas las categorías $(p \leq 0,01)$. En cambio, en el compuesto de primavera sólo las categorías en las que la cobertura arbórea es inferior al 40\% presentan diferencias estadísticamente significativas. De hecho, mientras que en los compuestos de verano y otoño las diferencias entre los valores promedio en las categorías extremas $(<10 \%$ y $>60 \%)$ adquieren cierta significación $\left(\sim 2,5^{\circ} \mathrm{C}\right)$, en el compuesto de primavera apenas alcanzan $1^{\circ} \mathrm{C}$ cada incremento del 10\% en la cobertura arbórea.

Teniendo en cuenta el carácter perennifolio de Quercus ilex, la especie arbórea con mayor representación espacial en la dehesa de Cáceres, es precisamente la diferente contribución del estrato herbáceo y su variabilidad fenológica interanual el principal factor explicativo de la variabilidad espacial de la Ts en los diferentes compuestos. Con- 


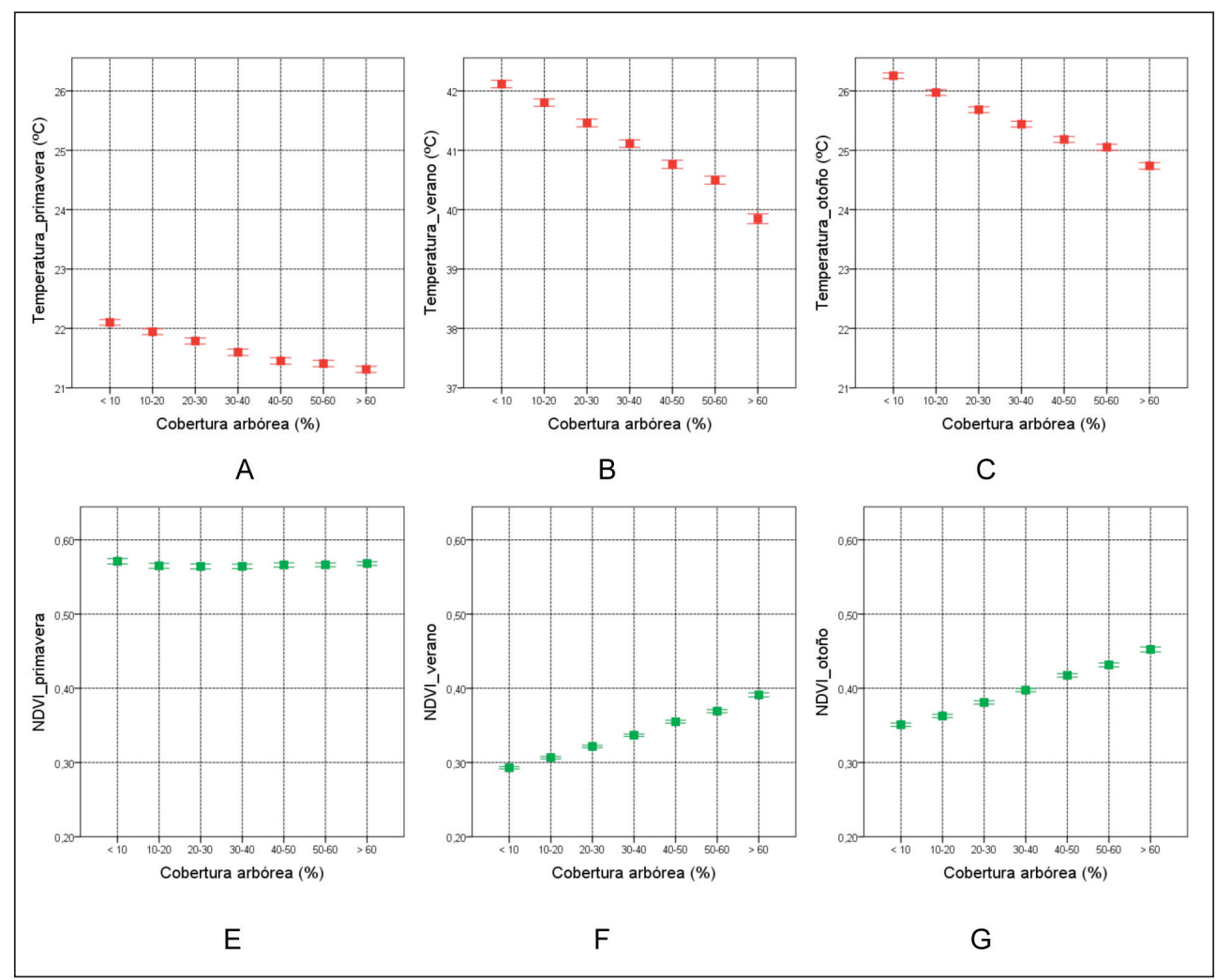

Figura 5. Distribución de la Ts (A-C) y NDVI (D-F) por categorías de dehesa (según intervalos de cobertura arbórea) en primavera (izquierda), verano (centro) y otoño (derecha). Las barras indican el intervalo de confianza 95\% para el valor medio.

cretamente, los procesos de senescencia del estrato herbáceo durante el verano y el incremento de la contribución del suelo desnudo a la radiación emitida explicarían el mayor contraste térmico que se produce entre diferentes categorías de densidad de arbolado. Además, la menor presencia de zonas con sombra en los píxeles con escaso arbolado también contribuye de manera positiva en el incremento de la Ts. En términos generales, en verano una disminución del 10\% en la cobertura arbórea (con el consiguiente aumento porcentual de la cobertura de pastizal) se asocia con un aumento de $0,4^{\circ} \mathrm{C}$ en la Ts. En cambio, en primavera, cuando el estrato herbáceo se sitúa fisiológicamente en su máximo productivo, especialmente a finales de abril (San Miguel, 2009), se produce una reducción en la exposición de suelo desnudo y, sobre todo, un incremento de los procesos de transpiración que explicarían la disminución de gradientes térmicos entre categorías de dehesa. En otoño, a pesar de existir un máximo secundario en la producción de biomasa a principios de noviembre (Moreno y Pulido, 
2009), se recoge un patrón de distribución semejante al de verano, aunque los contrastes térmicos entre densidades de arbolado son más moderadas. En este caso se aprecia un decremento de $0,25^{\circ} \mathrm{C}$ por cada incremento del $10 \%$ en la cobertura arbórea.

Por tanto, son las variaciones fisiológicas del estrato herbáceo, complemento del arbóreo en los sistemas de dehesa, las que parecen explicar en gran medida los patrones de distribución espacial de la Ts. El análisis de la distribución de los valores de NDVI en las categorías de dehesa permite comprobar este extremo (Tabla 3, Figura 5 D-F). El NDVI, al ser un índice espectral muy correlacionado con variables biofísicas como la actividad clorofílica, la densidad y el vigor de la vegetación, presenta valores muy homogéneos (valor medios 0,57, Tabla 2) en el compuesto de primavera independientemente de la densidad del arbolado. De este modo, no se recogen diferencias significativas entre las categorías ( $\mathrm{p}>0,05)$. Por el contrario, en los compuestos de otoño

Tabla 3. Estadísticos descriptivos de NDVI por categorías de dehesa y estaciones climáticas

\begin{tabular}{|c|c|c|c|c|c|}
\hline & Categoria (\% de cobertura arbórea) & Media & Desviación típica & Mínimo & Máximo \\
\hline \multirow{7}{*}{  } & $<10$ & 0.57 & 0.06 & 0.24 & 0.71 \\
\hline & $10-20$ & 0.56 & 0.05 & 0.33 & 0.69 \\
\hline & $20-30$ & 0.56 & 0.05 & 0.31 & 0.72 \\
\hline & $30-40$ & 0.56 & 0.05 & 0.39 & 0.69 \\
\hline & $40-50$ & 0.57 & 0.05 & 0.37 & 0.70 \\
\hline & $50-60$ & 0.57 & 0.04 & 0.35 & 0.69 \\
\hline & $>60$ & 0.57 & 0.04 & 0.40 & 0.67 \\
\hline \multirow{7}{*}{  } & $<10$ & 0.29 & 0.03 & 0.20 & 0.39 \\
\hline & $10-20$ & 0.31 & 0.02 & 0.23 & 0.39 \\
\hline & $20-30$ & 0.32 & 0.03 & 0.23 & 0.46 \\
\hline & $30-40$ & 0.34 & 0.03 & 0.25 & 0.45 \\
\hline & $40-50$ & 0.35 & 0.03 & 0.23 & 0.47 \\
\hline & $50-60$ & 0.37 & 0.03 & 0.18 & 0.52 \\
\hline & $>60$ & 0.39 & 0.04 & 0.28 & 0.55 \\
\hline \multirow{7}{*}{ 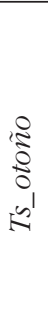 } & $<10$ & 0.35 & 0.04 & 0.23 & 0.48 \\
\hline & $10-20$ & 0.36 & 0.04 & 0.24 & 0.49 \\
\hline & $20-30$ & 0.38 & 0.04 & 0.27 & 0.51 \\
\hline & $30-40$ & 0.40 & 0.03 & 0.27 & 0.51 \\
\hline & $40-50$ & 0.42 & 0.04 & 0.26 & 0.55 \\
\hline & $50-60$ & 0.43 & 0.04 & 0.27 & 0.59 \\
\hline & $>60$ & 0.45 & 0.05 & 0.30 & 0.62 \\
\hline
\end{tabular}


y, especialmente, en el verano, se recogen diferencias significativas en los valores de NDVI entre categorías de arbolado. Los niveles de NDVI más altos se observan en la categoría con el máximo porcentaje de cobertura arbórea (>60\%) disminuyendo en forma progresiva hasta llegar a los niveles más bajos en las categorías con predominio de pastizal, lo que demuestra un patrón de distribución completamente diferente (aunque relacionado) al presentado por la Ts.

Los diagramas de dispersión de la figura 6 representan las relaciones, píxel a píxel, entre los valores promedio de la Ts y los promedios de NDVI en los compuestos de primavera (6A), verano (6B) y otoño (6C). El color de los puntos hace referencia a los porcentajes de cobertura arbórea.

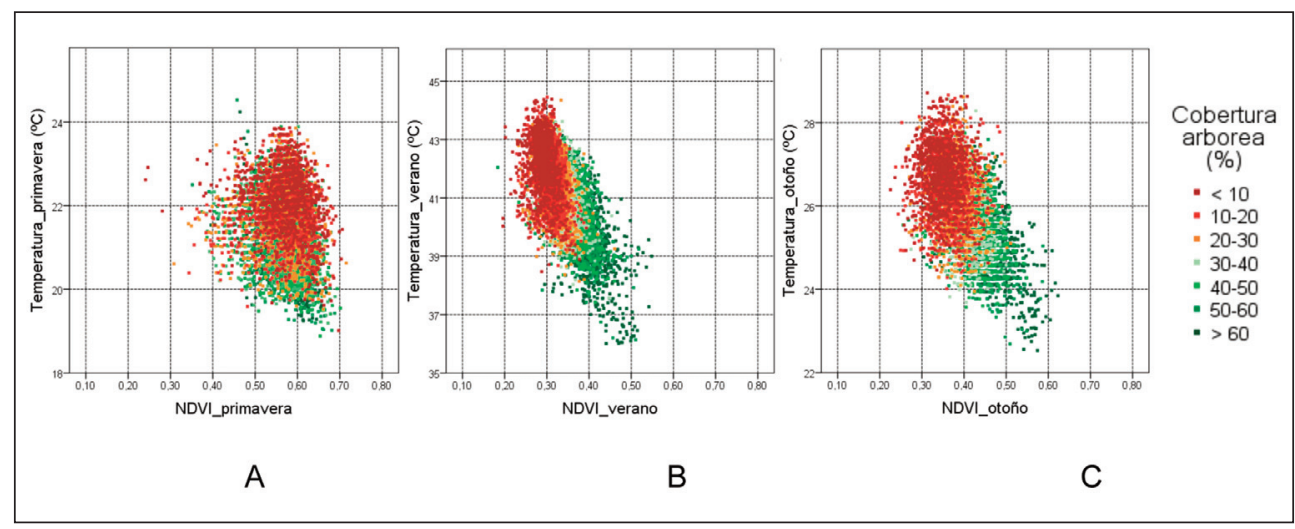

Figura 6. Diagramas de dispersión de NDVI y Ts en primavera (A), verano (B) y otoño (C).

De acuerdo con la interpretación del espacio formado por las variables NDVI-Ts sugerida por Jiang e Islam (2001), los puntos correspondientes a zonas de suelo desnudo se localizarían en el extremo superior izquierdo, lugares donde la fracción leñosa es muy baja; en cambio, los puntos correspondientes a los píxeles completamente cubiertos por vegetación se localizarían en el extremo inferior derecho.

De esta manera, en los diagramas de los compuestos de verano y otoño se advierte una relación negativa entre los valores de NDVI y Ts y una organización de los píxeles acorde a los parámetros preestablecidos. Sin embargo, en el compuesto de primavera esta relación es inexistente y las diferentes clases de arbolado se solapan en el espacio del diagrama NDVI-Ts generando una distribución más homogénea en la que los píxeles rojos, correspondientes a las categorías con porcentajes inferiores al 10\% de cobertura arbórea, aparecen en toda la nube de puntos. Esto es debido a que en esta fecha la vegetación muestra una elevada actividad fotosintética, independientemente de la proporción del estrato arbóreo o del pastizal. 


\section{Conclusiones}

El análisis cartográfico de los polígonos de dehesa (obtenidos a partir de SIOSE) mediante ortofotografía de alta resolución espacial permite evaluar la heterogeneidad de los niveles de cubrimiento del arbolado dentro de los ecosistemas de dehesa, proporcionando un mayor nivel de precisión en el análisis de las relaciones con la Ts.

Se analizan los patrones espacio-temporales de la Ts estimada a partir de la serie de imágenes Landsat-5 TM del período 2009-2011 y su relación con las categorías de dehesa. La distribución espacial de la Ts está estrechamente relacionada con la cobertura de la superficie por la vegetación: la Ts más baja se observa en las áreas con mayor cobertura de la vegetación y la más elevada en las zonas de vegetación senescente o suelo desnudo. Por esa razón, en el ecosistema de dehesa el patrón espacial de Ts depende del ciclo fenológico del pastizal, que es el componente más dinámico. El ciclo de desarrollo del estrato herbáceo contiene momentos de intenso vigor en la primavera, así como estado de senescencia en verano. Esto explica la relación negativa que existe entre la cobertura arbórea y la Ts. Esta relación es más pronunciada en verano (diferencia entre los promedios de las categorías extremas $(<10 \%$ y $>60 \%)$ se acerca a $2,5^{\circ} \mathrm{C}$ ) debido al estado senescente de las herbáceas y a la menor importancia de las sombras proyectadas por el estrato arbóreo, observándose un aumento de Ts de $0,4^{\circ} \mathrm{C}$ con cada disminución de 10\% en la cobertura arbórea. Las diferencias en la Ts entre los tipos de dehesa se mantienen en primavera, aunque son de menor magnitud $\left(\sim 1^{\circ} \mathrm{C}\right)$ por cuanto la vegetación de los dos estratos de la dehesa (arbóreo y pastizal) está en el máximo vigor y el promedio de las diferencias en Ts con la disminución de 10\% en la cobertura arbórea se reduce a $0,1^{\circ} \mathrm{C}$.

El estudio en su conjunto responde a la necesidad de generar información que pueda ser integrada en los modelos de respuesta del ecosistema de dehesa a los efectos del cambio climático (Moreno y Pulido, 2009; Joffre et al., 1999). En este contexto, se ha demostrado la capacidad de los sensores remotos para monitorizar la variabilidad espacio-temporal de la temperatura de la superficie (Ts), una de las variables destacadas en otras investigaciones (a.o. Plieninger et al., 2004), y contribuir así a la definición de estrategias para mantener la sostenibilidad de este ecosistema.

\section{Agradecimientos}

Este trabajo se ha realizado en el marco del proyecto FLUXPEC (CGL2012-34383) financiado por el Ministerio de Economía y Competitividad. Los autores también agradecen la financiación de la investigación predoctoral de Lidia Vlassova por parte de SENESCYT-Ecuador. 


\section{Bibliografía}

Baldocchi, D.D., Xu, L., Kiang, N., 2004. How plant functional-type, weather, seasonal drought, and soil physical properties alter water and energy fluxes of an oak-grass savanna and an annual grassland. Agricultural and Forest Meteorology 123, pp. 13-39.

Blanco, E., Casado, M.A., Costa, M., Escribano, R., García, M., Génova, M., Gómez, A., Gómez, F., Moreno, J.C., Morla, C., 1997. Los bosques ibéricos. Una interpretación geobotánica. Planeta, Barcelona.

Carreiras, J., Pereira, J.M., Pereira, J.S., 2006. Estimation of tree canopy cover in evergreen oak woodlands using remote sensing. Forest ecology and management 123, pp. 45-53.

Chander, G., Markham, B.L., Helder, D.L., 2009. Summary of current radiometric calibration coefficients for Landsat MSS, TM, ETM+, and EO-1 ALI sensors. Remote sensing of environment 113, pp. 893-903.

Daughtry, C.S.T., Walthall, C.L., Kim, M.S., De Colstoun, E.B., McMurtrey, J.E., 2000. Estimating corn leaf chlorophyll concentration from leaf and canopy reflectance. Remote Sensing of Environment 74(2), pp. 229-239.

Devesa, J.A., 1995. Vegetación y flora de Extremadura. Universitas, Badajoz.

Díaz, M., Campos P., Pulido F.J., 1997. Spanish Dehesas: a diversity in land-use and wildlife. En: Pain D.J., Pienkowski M.W. (Eds). Farming and birds in Europe. Academic, London.

Eagleson, P.S., Tellers, T.E., 1982. Ecological optimality in water limited natural soil vegetation systems. Water Resources Research 18, pp. 341-354.

Hwang, T., Song, C., Bolstad, P.V., Band, L.E., 2011. Downscaling real-time vegetation dynamics by fusing multi-temporal MODIS and Landsat NDVI in topographically complex terrain. Remote Sensing of Environment 115(10), pp. 2499-2512.

Huntsinger, L., Campos, P., Starrs, P.F., Oviedo, J.L., Díaz, M., Standiford, R.B., Montero, G., 2013. Working Landscapes of the Spanish Dehesa and the California Oak Woodlands: An Introduction. En: P. Campos, L. Huntsinger, J.L. Oviedo, P.F. Starrs, M. Diaz, R. Standiford y G. Montero (Eds.) Mediterranean oak woodland working landscapes. Springer, Netherlands.

Jiang, L., Islam, S., 2001. Estimation of surface evaporation map over southern Great Plains using remote sensing data. Water Resources Research 37(2), pp. 329-340.

Jiménez-Muñoz, J.C., Sobrino, J.A., Cristobal, J., Soria, G., Niyerola, M., Pons, X., Hidalgo, V., Franch, B., Romaguera, M., Oltra-Carrió, R., Julien, Y., Mattar, C., 2010. Obtención de la temperatura de la superficie terrestre a partir de la serie histórica de LANDSAT. Revista de Teledetección 33, pp. 53-63.

Joffre, R., Rambal, S., Ratte, J.-P., 1999. The dehesa system of southern Spain and Portugal as a natural ecosystem mimic. Agroforestry systems 45, pp. 57-79.

Miquelajauregui, Y., 2013. Modelos de simulación de la dinámica del carbono. En J.A. Blanco (Ed.). Aplicaciones de modelos ecológicos a la gestón de recursos naturales. Barcelona, OmniaScience.

Montero, G., San Miguel, A., Cañellas, I., 1998. System of Mediterranean silviculture La Dehesa. En R.M. Jiménez Díaz, J. Lamo de Espinos (Eds.) Agricultura Sostenible. Mundi Prensa, Madrid. 
Moreno, G., Pulido, F., 2009. The functioning, management and persistence of dehesas. En A. Rigueiro-Rodríguez, J. McAdam, M.R. Mosquera-Losada (Eds.). Agroforestry in Europe, pp. 127160. Springer.

Olea, L., López-Bellido, R.J., Poblaciones, M.J., 2005. Europe types of silvopastoral systems in the Mediterranean area: dehesa. En M.R. Mosquera, A. Rigueiro, J. McAdam (Eds.). Silvopastoralism and Sustainable Land Management. CABI Publishing.

Plieninger, T., Pulido, F.J., Schaich, H., 2004. Effects of land-use and landscape structure on holm oak recruitment and regeneration at farm level in Quercus ilex L. Dehesas. Journal of Arid Environments 57, pp. 345-364.

Pulido, F.J., Diaz, M., de Trucios, S.J.H., 2001. Size structure and regeneration of Spanish holm oak Quercus ilex forests and dehesas: effects of agroforestry use on their long-term sustainability. Forest Ecology and Management 146, pp. 1-13.

Pulido, F., Picardo, A. (Coords.), 2010. Libro verde de la dehesa. Documento para el debate hacia una Estrategia Ibérica de gestión. Recuperado el 1 de octubre de 2015, de http://www. pfcyl.es/documento/libro-verde-de-la-dehesa

Quattrochi, D.A., Luvall, J.C., 2000. Thermal remote sensing in land surface processes. CRC Press.

Rodriguez-Iturbe, I., D’Odorico, P., Porporato, A., Ridolfi, L., 1999. On the spatial and temporal links between vegetation, climate, and soil moisture. Water Resources 35 (12), pp. 3709-3722.

Rouse, J.W., Haas, R.W., Shell, J.A., Deering, D.W., Harlan, J.C., 1974. Monitoring the vernal advancement and retrogradation of natural vegetation. NASA/GSFCT Type III final report. Greenbelt, EUA.

San Miguel, A., 1994. La dehesa española. Origen, tipología, características y gestión. Fundación Conde del Valle de Salazar, Madrid.

San Miguel, A. (Coord.), 2009. Los pastos de la comunidad de Madrid. Tipología, cartografía y evaluación. Comunidad de Madrid, Madrid.

Sobrino, J.A., Raissouni, N., 2000. Toward remote sensing methods for land cover dynamic monitoring: Application to Morocco. International Journal of Remote Sensing 2, pp. 353-366.

Tamhane, A.C., 1977. Multiple comparisons in model I one-way ANOVA with unequal variances. Communications in Statistics Series B 9, pp. 167-178.

Turner, D.P., Cohen, W.B., Kennedy, R.E., Fassnacht, K.S. \& Briggs, J.M., 1999. Relationships between leaf area index and Landsat TM spectral vegetation indices across three temperate zone sites. Remote Sensing of Environment 70(1), pp. 52-68.

Vlassova, L., Pérez-Cabello, F., Mimbrero, M., Llovería, R., García-Martín, A., 2014. Analysis of the Relationship between Land Surface Temperature and Wildfire Severity in a Series of Landsat Images. Remote Sensing 6(7), pp. 6136-6162.

Volder, A., Briske, D.D., Tjoelker, M.G., 2013. Climate warming and precipitation redistribution modify tree-grass interactions and tree species establishment in a warm temperate savanna. Global Change Biology 19, pp. 843-857.

Weng, Q., Lu, D., Schubring, J., 2004. Estimation of land surface temperature-vegetation abundance relationship for urban heat island studies. Remote Sensing of Environment 89(4), pp. 467-483. 
Wolfe, R., Masek, J., Saleous, N., Hall, F., 2004. LEDAPS: mapping North American disturbance from the Landsat record. En: Proceedings of the IEEE International Geoscience and Remote Sensing Symposium, September 19-26, 2004. Anchorage, Alaska, EUA.

Zeng, N., Neelin, J.D., 2000. The role of vegetation-climate interaction and interannual variability in shaping the African savanna. J. Clim. 13, pp. 2665-2669. 